\title{
BCS Model in Tsallis' Statistical Framework
}

\author{
Lizardo H. C. M. Nunes and E. V. L. de Mello \\ Departamento de Física, Universidade Federal Fluminense, Av. Litorânea s/n, \\ Boa Viagem Niterói, Rio de Janeiro, 24210-340, Brazil
}

\begin{abstract}
We show that there is an effect of nonextensivity acting upon the BCS model for superconductors in the ground state that motivates its study in the Tsallis' statistical framework. We show that the weak-coupling limit superconductors are well described by $q \sim 1$, where $q$ is a real parameter which characterizes the degree of nonextensivity of the Tsallis' entropy. Nevertheless, small deviations with respect to $q=1$ provide better agreement when compared with experimental results. To illustrate this point, making use of an approximated Fermi function, we show that measurements of the specific heat, ultrasonic attenuation and tunneling experiments for tin (Sn) are better described with $q=0.99$.
\end{abstract}

Key words: BCS theory, Nonextensive Statistical Mechanics

PACS: $74.20 . \mathrm{Fg}, 05.90 .+\mathrm{m}$

\section{Introduction}

About 10 years ago, Tsallis[1] proposed the following entropic form:

$$
S_{q}=-k_{B} \frac{1-\operatorname{Tr} \rho^{q}}{1-q},
$$

where $k_{B}$ is a positive constant, $\rho$ is the matrix density of the system and $q$ is a real parameter. The thermodynamics constructed from (1) has been applied to several physical systems in the last years. Indeed, nonextensive statistics was used to investigate physical systems which presented nonextensive features. Amongst them, there are stellar polytrops[3], solar neutrino problem[4], galaxy clusters[5], etc. It should be remarked that the formalism established above

Email address: lizardo@if.uff.br (Lizardo H. C. M. Nunes ). 
seems to be appropriate particularly to systems interacting via long-range interactions.

Furthermore, formal results were achieved and techniques were developed. Indeed, from the properties of the Tsallis' entropy, Plastino and Tsallis[6] generalized the Boguljubov inequalities and justified the variational method; also, this nonextensive thermodynamics preserves the Legendre Transform formalism[7,11]. It's also possible to establish a relation between the partition function with $q=1$, as usually calculated by the standard statistics, and the generalized $q \neq 1$ nonextensive partition function[8] (for this purpose one use the Hilhorst integral ). This result was crucial to the generalization of the Green's functions method $[9,10]$, which is a useful tool in the discussion of a many-body system for any possible real value for $q$, where $q$ measures the nonextensivity of the system.

In this article study the BCS (Bardeen, Cooper and Schrieffer) model in Tsallis' statistical framework. Indeed, the BCS theory for superconductivity describes very well, at least qualitatively, a vast amount of experimental results for a wide range of materials; these materials, henceforth, will be called usual superconductors, since they are well explained by the BCS theory. Assuming that the exchange of phonons between electrons is responsible for the superconducting phenomena, we shall also assume that the criterium for the appearance of superconductivity is that the Coulomb repulsion is surmounted by the effective attraction caused by the phononic interaction. Instead of calculating the interactions involved by "first principles" (divergences in the perturbative chain postponed for years the development of the theory) BCS simply considered a mean-field approach with excellent results. Such improved theory will not be considered in this article. In fact, our aim is to develop a $q$-dependent formalism which may be applied to materials or a class of materials which systematically deviates from the usual theory. As a first approach, we will concern ourselves to the weak-coupled limit of the BCS theory, but the strong coupling limit and the generalization of the theory to other potentials with the correlations included, for instance, is already under study and will be subject of further publication.

In next section we show that there is an effect of nonextensivity acting upon the BCS model at $T=0$, then we generalize the well known gap equation and use it to calculate quantities that will be compared with the experimental results for the usual superconductors. 


\section{Motivation}

Consider the well known "reduced" Hamiltonian proposed by BCS and used to study the appearance of superconductivity in simple metals [14],

$$
H_{\text {red }}=\sum_{k} \epsilon_{k}\left(n_{k}+n_{-k}\right)+\sum_{k k^{\prime}} V_{k k^{\prime}} b_{k^{\prime}}^{\dagger} b_{k}
$$

where the operator $b_{k}^{\dagger}=c_{k}^{\dagger} c_{-k}^{\dagger}$ creates a Cooper pair in the singlet state and the model describes interacting electrons forming singlet Cooper pairs. This assumption may be justified in terms of an instability of the Fermi sea being caused by binding of zero-momentum pairs. The only relevant electrons to the superconducting state are those in a small neighborhood of the Fermi level within a layer twice the Debye frequency thick (If one consider electron-phonon interaction as the sole responsible for the superconductivity phenomena.). Notice that we have used the convention that an explicitly negative $k$ has down spin and vice versa.

The transformed Hamiltonian, in a semi-classical view, has its unperturbed Fermi sea characterized by "spins" aligned only in the $\hat{z}$ direction; once the phononic attraction between electrons is present, a non-null component of $\overrightarrow{S_{k}}$ perpendicular to $\hat{z}$ shows up and this configuration is determined in terms of the orientation $S_{x k} / S_{z k}=\tan \theta_{k}$. Indeed, following Bogoliubov's notation[16], $\sin \theta_{k}=u_{k} v_{k}$ and this quantity is related to the superconducting gap energy, $\Delta_{k}$, this gap may be seen as the order parameter of the phase transition superconductor-normal $\left(\Delta_{k} \neq 0\right.$ represents superconducting solutions.).

Following the prescription briefly introduced above, the system may be analyzed not at an arbitrary temperature $T$ below the transition temperature $T_{c}$, but particularly at $T=0$, where all relevant electrons are in the condensate state, henceforth, paired. When the temperatures increases, the possible excitations of the system are the quasi-particles and the excited pairs. As pointed out by Schrieffer[17], at the zero-temperature the operator $n_{k}+n_{-k}$ may be replaced by $2 b_{k}^{\dagger} b_{k}$, twice the occupation number of pairs. Hence, we have to work in the grand-canonical ensemble adding to (2) the operator $-\mu N$, where $N$ is $\sum_{k} n_{k}+n_{-k}$ and the chemical potential, $\mu$, may be regarded as essentially the Fermi energy[18]. Therefore, we get a modeled system of interacting electrons with net energy $\epsilon_{k}-\mu$, what is consistent with the picture of only relevant electrons for superconductivity possessing energies in the vicinity of the Fermi level.

In order to study the attractive potential, we perform a Fourier transform of (2) assuming an isotropic superconductor. The 1/2 "spin" operators are defined by 


$$
\begin{aligned}
& b_{i}^{\dagger}=S_{i x}+i S_{i y}=S_{i}^{+} \\
& b_{j}=S_{j x}-i S_{j y}=S_{j}^{-} .
\end{aligned}
$$

After writing the "reduced" Hamiltonian at $T=0$ in terms of these pseudo spins $(3,4)$, it is possible to show that the model is isomorphous to the $X Y$ model:

$$
\mathbf{H}_{r e d}=\sum_{i j}\left(t_{i j}+V_{i j}\right) b_{i}^{\dagger} b_{j}
$$

where

$$
t_{i j}=\frac{2}{\Omega} \sum_{k}\left(\epsilon_{k}-\mu\right) e^{-i \mathbf{k}\left(\mathbf{r}_{\mathbf{i}}-\mathbf{r}_{\mathbf{j}}\right)}
$$

and

$$
V_{i j}=\frac{2}{\Omega} \sum_{k k^{\prime}} V_{k k^{\prime}} e^{-i\left(\mathbf{k r}_{\mathbf{i}}-\mathbf{k}^{\prime} \mathbf{r}_{\mathbf{j}}\right)}
$$

with $\Omega$ being a normalizing constant.

As pointed out by Tsallis and Anteneodo [19], this classical Hamiltonian may be studied with $V_{i j} \propto r_{i j}^{-\alpha}$ as the coupling between sites, where $r_{i j}=r_{i}-r_{j}$ and $\alpha$ is the range of the interaction essentially. A typical quantity that should be calculated at $T=0$ is $\int_{1}^{\infty} d r r^{d-1} r^{-\alpha}$, which is related to the internal energy per particle. If $0 \leq \alpha \leq d$, It's a trivial task to show that the internal energy per particle diverges in the thermodynamic limit, what was called weak violation of Boltzmann-Gibbs (BG) statistics (in contrast to "strong" $T \neq 0$ violation). This result also reveals the role played by the range and the dimension of the system in the scenario of the nonextensivity.

Following the path established above and assuming $d=3$, we will show that there is a nonextensive effect acting upon the BCS model at $T=0$. For this purpose we will use the well known BCS mean-field interaction

$$
V_{k k^{\prime}}=\left\{\begin{array}{l}
-V, \text { we }\left|\epsilon_{k}-\mu\right|,\left|\epsilon_{k^{\prime}}-\mu\right|<\hbar w_{D} \\
0, \text { otherwise }
\end{array}\right.
$$

where $\hbar w_{D}=k_{B} \theta_{D}$ and $\theta_{D}$ is the Debye temperature. The spatial dependence of this interaction will be found approximating the sums over $k$ and $k^{\prime}$ in (7) by volume integrals. The region of integrability, of course, will be constrained to values of $\mathbf{k}$ related to the relevant electrons only: $\alpha_{D} \equiv \sqrt{\frac{2 m_{e} w_{D}}{\hbar}}>|| \mathbf{k}\left|-k_{F}\right|$, 
where $m_{e}$ is the electron mass, what defines a neighborhood around $k_{F}$. Hence, we finally get

$$
V_{i j}=\frac{(8 \pi)^{2}}{\Omega} v\left(r_{\beta}\right)
$$

with

$$
\begin{aligned}
v\left(r_{\beta}\right)= & \frac{1}{r_{\beta}^{2}}\left[\frac{\cos \left(k_{F} r_{\beta}\right) \sin \left(\alpha_{D} r_{\beta}\right)}{r_{\beta}}-\right. \\
& \left.\alpha_{D} \cos \left(\alpha_{D} r_{\beta}\right) \cos \left(k_{F} r_{\beta}\right)+k_{F} \sin \left(k_{F} r_{\beta}\right) \sin \left(\alpha_{D} r_{\beta}\right)\right]
\end{aligned}
$$

and $\beta=i, j$ indistinctly (Notice $r_{\beta}=\left|\mathbf{r}_{\beta}\right|$ ).

There's a one-to-one correspondence between the $i$ 's lattice sites and the pairs at $T=0$, so it's reasonable to replace sums over $i$ and $j$ by integrals. Again, we get a product of identical integrals and the potential energy is proportional to $\left[\int d^{3} r v_{\beta}\right]^{2}$. In agreement of what is expected for a extensive system, the first part of the above integral converges to a constant factor in the thermodynamic limit ( since $k_{F}, a_{D}>0$ and $\left.\lim _{N \rightarrow \infty} \int d^{3} r\left(\cos \left(k_{F} r\right) \sin \left(\alpha_{D} r\right) / r^{3}\right)=\pi / 2\right)$. However, the second and third contribution give raise to oscillatory fluctuations in the potential energy according to the number of pairs in the system:

$$
\begin{aligned}
& \int d^{3} r \frac{1}{r^{2}}\left[k_{F} \sin \left(k_{F} r\right) \sin \left(\alpha_{D} r\right)-\alpha_{D} \cos \left(\alpha_{D} r\right) \cos \left(k_{F} r\right)\right]= \\
& 4 \pi\left\{\sin \left[\left(k_{F}+\alpha_{D}\right) N\right]-\sin \left[\left(k_{F}-\alpha_{D}\right) N\right]\right\}
\end{aligned}
$$

These fluctuations are bounded but still present even for a very large number of pairs and they don't decrease as you add electrons to the system. Further, the energy cannot be expressed as the energy per particle times the number of particles itself, as commonly done for extensive systems.

Therefore, we conclude that there is a formal nonextensive effect acting upon the $B C S$ model at $T=0$ as mentioned above. The nonextensive character may be regarded as a motivation for the analysis of the BCS theory in Tsallis' statistical framework.

\section{The Method}

In this section we will generalize the BCS gap equation in the Tsallis' statistical framework making use of the well known Gorkov's anomalous functions. Also, 
it's that the lifetime of the quasi-particles is infinity, since the interaction is time-independent.

Originally, BCS proposed a wave function for the ground state, $\left|\psi_{0}\right\rangle$, describing $N$ paired electrons. This wave function was given in terms of a variational parameter that should be found minimizing the Helmholtz free energy. Regarding $\mathrm{BCS}$, the superconducting gap energy, $\Delta$, may be considered the order parameter of the phase transition. Notice that even before the full development of the theory this gap energy was observed in several experiments such as specific heat, ultrasonic attenuation rate, tunneling measurements, etc. The self-consistent equation for the superconducting gap was given by BCS[14] in a Hartree-Fock approximation and may be written as $\Delta_{k}=-\sum_{k k^{\prime}} V_{k k^{\prime}}\left\langle b_{k^{\prime}}^{\dagger}\right\rangle$, where $\left\langle b_{k}^{\dagger}\right\rangle$ is the expectation value in the BG statistics for the temperature dependent creation operator of a Cooper pair. A generalized version of this quantity, which includes Tsallis statistics and the $q$-dependent expectation value, shall simply be

$$
\Delta_{k}=-\sum_{k k^{\prime}} V_{k k^{\prime}}\left\langle b_{k^{\prime}}^{\dagger}\right\rangle_{q}
$$

Through a canonical transformation the system may be simply described by quasi-particles with a given dispersion relation[16] In order to calculate the $q$-expectation value found in (12) we define, for $q=1$ and except for a multiplicative factor, the propagator $G_{\sigma}(\mathbf{k}, \tau)=\left\langle T\left[c_{k \sigma}(\tau) c_{k \sigma}^{\dagger}(0)\right]\right\rangle$, where $T$ is the temporal ordering operator and $\hbar=1$ from now on. The propagator can be rewritten as $G_{\sigma}(\mathbf{k}, \tau)=\theta(\tau)\left\langle c_{k \sigma}(\tau) c_{k \sigma}^{\dagger}(0)\right\rangle-\theta(-\tau)\left\langle c_{k \sigma}^{\dagger}(0) c_{k \sigma}(\tau)\right\rangle$ in a fermionic system. In order to calculate $G$ in interacting systems we may use two methods mainly: Feynman diagrams and the construction of the equation of moment. We attempt to generalize the latter by closely following the development discussed in $\operatorname{Ref}[20]$.

Consider the $\operatorname{advanced}(a)$ and $\operatorname{retarded}(r)$ double-time Green's functions $[21,9]$ :

$$
\begin{gathered}
G_{A B}^{(r, q)}=\theta\left(t-t^{\prime}\right)\left\langle\left[\mathbf{A}(t), \mathbf{B}\left(t^{\prime}\right)\right]_{\eta}\right\rangle_{q} \\
G_{A B}^{(a, q)}=-\theta\left(t-t^{\prime}\right)\left\langle\left[\mathbf{A}(t), \mathbf{B}\left(t^{\prime}\right)\right]_{\eta}\right\rangle_{q}
\end{gathered}
$$

where $\theta(\tau)$ is the characteristic function times an arbitrary multiplying factor. By definition, $\left\langle[\mathbf{A}, \mathbf{B}]_{\eta}\right\rangle_{q}=\langle\mathbf{A B}+\eta \mathbf{B A}\rangle_{q}$, with $\eta=-1$; if $\mathbf{A}$ and $\mathbf{B}$ are bosonic operators, and $\eta=1$ if fermionic. The Green's functions admits a Fourier series expansion with Fourier transform

$$
\ll A, B \gg_{w}^{(j, q)}=\frac{1}{2 \pi} \int_{-\infty}^{\infty} d \tau G_{A B}^{(j, q)}\left(t, t^{\prime}\right) e^{i w \tau}
$$


where $j=r, a$ and $\tau=t-t^{\prime}$. Assuming that $\left[\mathbf{A}(\mathbf{t}), \mathbf{B}\left(\mathbf{t}^{\prime}\right)\right]_{\eta}$ is a non null operator, the Green's function can be represented by means of the Hilhorst integration $[9,8]$, which relates the $q$-expectation value of observables to its standard $q=1$ analogous average in the BG statistics. The correlation function is given then by:

$$
\begin{aligned}
& \left\langle\mathbf{B}\left(t^{\prime}\right) A(t)\right\rangle_{q}= \\
& \frac{1}{\mathcal{Z}_{q}} \int_{\mathcal{C}} d \xi K_{q}(\xi) \mathcal{Z}_{1}((q-1) \xi \bar{\beta}) \times \\
& \int_{-\infty}^{\infty} d w \frac{\ll A, B \gg_{w+i 0^{+}}^{(1)} \ll A, B \gg_{w-i 0^{+}}^{(1)}}{e^{(q-1) \xi \bar{\beta} w}+\eta}
\end{aligned}
$$

where the kernel $K_{q}(\xi)$ is a $q$-dependent function presented in the integral representation of the Green's function, according Lenzi's notation[10].

We shall calculate the equation of motion of each propagator of interest regarding the phenomena of superconductivity and than calculate the gap energy, in order to compare it with experimental measurements.

In a time-independent Hamiltonian (such as the case) the corresponding Green's functions depend only upon $t-t^{\prime}=\tau$. The equation of motion for $\ll c_{k \uparrow}, c_{k \uparrow}^{\dagger} \gg_{w}^{(1)}$, is:

$$
\left(w-\gamma_{k}\right) \ll c_{k \uparrow}, c_{k \uparrow}^{\dagger} \gg_{w}^{(1)}=\frac{i}{2 \pi}+\Delta \ll c_{-k \downarrow}^{\dagger}, c_{k \uparrow}^{\dagger} \gg_{w}^{(1)},
$$

where $\gamma_{k}=\epsilon_{k}-\mu$. For $\ll c_{-k \downarrow}^{\dagger} c_{k \uparrow}^{\dagger}, \gg_{w}^{(1)}$, the procedure is identical and

$$
\left(w+\gamma_{k}\right) \ll c_{-k \downarrow}^{\dagger}, c_{k \uparrow}^{\dagger} \gg_{w}^{(1)}=\Delta \ll c_{k \uparrow}^{\dagger}, c_{-k \uparrow} \gg_{w}^{(1)} .
$$

This is the Gorkov's anomalous function and it's strictly related to the superconducting phenomena, i. e., the appearance of Cooper pairs. Notice that the results so far obtained are known and are also restricted to the standard statistics.

Finally, the propagator $\ll c_{-k \downarrow}^{\dagger}, c_{k \uparrow}^{\dagger} \gg_{w}^{(1)}$ is

$$
\begin{aligned}
\ll c_{k \uparrow}^{\dagger}, c_{-k \downarrow}^{\dagger} & \gg_{w}^{(1)}=\left(\frac{i}{2 \pi}\right) \frac{\Delta}{w^{2}-\gamma_{k}^{2}-\Delta^{2}} \\
& =\left(\frac{i}{2 \pi}\right) \frac{\Delta}{2 E_{k}}\left[\frac{1}{w-E_{k}}-\frac{1}{w+E_{k}}\right]
\end{aligned}
$$


where $E_{k}=\sqrt{\left(\epsilon_{k}-\mu\right)^{2}+\Delta_{k}^{2}}$ is the energy of the quasi-particle. When $\Delta=$ 0 , we get $E_{k}=\epsilon_{k}-\mu$, which is the electron energy with respect to the chemical potential $\mu$ and, indeed, superconducting solutions are given by $\Delta \neq$ 0 . Furthermore, from $E_{k}$, one can easily realize that the minimum amount of energy to create an excitation in the superconducting state is $\Delta$.

Combining (16) and making use of the $\left(w-w^{\prime} \pm i 0^{+}\right)^{-1}=P\left(w-w^{\prime}\right)^{-1} \mp$ $i \pi \delta\left(w-w^{\prime}\right)$, one have

$$
\begin{aligned}
& \left\langle c_{-k \downarrow}^{\dagger}, c_{k \uparrow}^{\dagger}\right\rangle_{q}= \\
& -\frac{1}{\mathcal{Z}_{q}} \int_{\mathcal{C}} d \xi K_{q}(\xi) \mathcal{Z}_{1}((q-1) \xi \bar{\beta}) \frac{\Delta}{2 E_{k}} \int_{-\infty}^{\infty} d w \frac{\delta\left(w-E_{k}\right)-\delta\left(w+E_{k}\right)}{e^{(q-1) \xi \bar{\beta} w}+1}= \\
& -\frac{\Delta}{2 E_{k} \mathcal{Z}_{q}} \int_{\mathcal{C}} d \xi K_{q}(\xi) \mathcal{Z}_{1}((q-1) \xi \bar{\beta})\left[1-2 f_{1}\left((q-1) \xi \bar{\beta} E_{k}\right)\right] .
\end{aligned}
$$

One can easily realize that the whole $q$-dependence of the quantity above is contained in the generalized version of the Fermi distribution function, $f_{q}$, since $\mathcal{Z}_{q}^{-1} \int_{\mathcal{C}} d \xi K_{q}(\xi) \mathcal{Z}_{1}((q-1) \xi \bar{\beta})=1$ and $f_{q}$ can be also written in terms of its own integral representation.

Finally, the self-consistent equation for the superconducting gap equation is:

$$
\Delta=-\sum_{k} V_{k k^{\prime}}\left\langle b_{k}^{\dagger}\right\rangle_{q}=\sum_{k} V_{k k^{\prime}} \frac{\Delta}{2 E_{k}}\left[1-2 f_{q}\left(\beta E_{k}\right)\right]
$$

which is identical to that found by simply diagonalize the reduced Hamiltonian by a canonical variable transformation proposed by Bogoljubov[16] but with $f_{q}$ in the place of the usual Fermi function $f$.

In the case usual superconductors it is reasonable to assume that the $q$ involved in numerical calculations will not deviate appreciably from the unity, since most of their properties have fairly agreed with the BCS results in the standard statistics. Hence we may use in numerical calculations an approximated Fermi function[12],

$$
f_{q}=\frac{1}{\left(e_{q}^{-\beta \gamma_{k}}\right)^{-q}+1}
$$

where $e_{q}^{x} \equiv[1+(1-q) x]^{\frac{1}{1-q}}$ is the generalized $q$-exponential function. The distribution function above avoids the self consistency found in (16) and might be considered a useful tool for systems near the extensivity[13]. 
Following BCS, we replace the sum over $k$ by an integral over $\gamma$ and the density of states shall be simply written as $N(\gamma) \approx N\left(\epsilon_{F}\right)$ or $N(0)$. Hence, the generalized self-consistency condition is

$$
\frac{1}{N\left(\epsilon_{F}\right) V}=\int_{0}^{\hbar w_{D}} d \gamma \frac{1-2 f_{q}(\beta E)}{E}
$$

and one can estimate the gap energy in the weak-coupling limit assuming $T_{c} \ll \theta_{D}$ :

$$
\frac{\Delta(0)}{k_{B} T_{c}}=2 e^{-N_{q}}
$$

where

$$
N_{q}=2 \int_{0}^{\infty} d x \frac{d}{d x} f_{q}(x) \ln x
$$

since $[14] \Delta(0)=2 \hbar w_{D} e^{-1 / N\left(\epsilon_{F}\right) V}$. In the limit $q \rightarrow 1$ we get $\Delta(0) / k_{B} T_{c}=1.76$, as usual. If one calculate self-consistently the gap equation taking into account the experimental data for $T_{c}$ and $\theta_{D}$, one get that the coupling $N\left(\epsilon_{F}\right) V$ is much less than unity. Superconductors with larger ratio $T_{c} / \theta_{D}$ tend to have greater disagreement with the predictions of the BCS model in the usual statistics.

\section{Comparison with Experimental Results}

In general, usual superconductors exhibit exponential behavior in the low temperature regime for several macroscopic measurable functions. As we will see below, the BCS model with its constant energy gap for a $s$-wave superconductor $(l=0)$ display such behavior in the standard statistics. On the other hand, the so-called exotic superconductors present power law behavior and it is believed that different pairing states may be responsible for such behavior. One of the most interesting results of the present paper is that, even for $s$-wave pairing state, it is possible to find power law behavior for different functions in the $T \ll T_{c}$ regime once $q \neq 1$.

In this section we compare experimental results for specific heat, ultrasonic attenuation and tunneling experince for some usual superconductors with different theoretical predictions for $q=1$. We show that small deviations with respect to $q=1$ provide better agreement when confronted to data, even regarding the approximations involved in the calculations. Furthermore, we 
expect that the same $q$ may fit the avaiable data for any different experiments of a given material.

\subsection{Specific Heat}

In an unitary volume, the eletronic specific heat in the superconductor state is

$$
C_{e}^{s}=2 k_{B} \beta^{2} \sum_{k}\left(E_{k}^{2}+\frac{\beta}{2} \frac{d}{d \beta} \Delta^{2}\right)\left[-\frac{d}{d\left(\beta E_{k}\right)} f_{q}\left(\beta E_{k}\right)\right]
$$

which may be interpreted as the specific heat associated to the quasi-particles with energy $E_{k}$, added to the contribution of the temperature dependent gap. Notice that when $q \rightarrow 1,-\frac{d}{d x} f_{1}(x)=f_{1}(x)\left(1-f_{1}(x)\right)$ and one recover the usual expression for a superconductor in the BG statistics.

The "normalized" jump $\left(C_{e}^{s}-C_{e}^{n}\right) / C_{e}^{n}$ may be measured and compared with theoretical predictions. The BCS theory with standard statistics predicts the result of 1.43 for the specific heat jump, and usual superconductors exhibit some deviations from this value as it is shown on Table 4.1 for several materials. We calculated numerically $\left.\left(C_{e}^{s}-C_{e}^{n}\right) / C_{e}^{n}\right)_{T_{c}}$ as a function of $q$ and therefore we can estimate the pertinent value of $q$ for the superconductors listed. Notice that $C_{e}^{n}$ was considered as a constant parameter in order to give the value of 1.43 at $q=1$ for the specific heat jump. Notice, from Table 4.1, that the appropriate $q$-value for $\mathrm{Sn}$ is 0.995 . we shall see below that numerical calculations for Sn using $q \sim 0.99$ show better agreement with measurements for the specific heat in the low temperature regime, ultrasonic attenuation rate and tunneling experiences. The fact that the same $q$ is able to give, at least qualitatively, a better agreement with different measurements serves as motivation to further application of nonextensive statistics in order to explain the anomalous features presented in some superconductors.

Thus, according to the above discussion, we show the specific heat for Sn and $\mathrm{V}$ in Fig. 5. From Table 4.1, we expected that $q=0.995$ should reproduce better data for Sn than $q=1$. We see also that the specific heat measurements for vanadium and tin [26] are well fitted by the exponential function $9.17 e^{-1.5\left(T_{c} / T\right)}$ in the temperature region $0.25<T / T_{c}<0.7$, which is quite remarkable if compared to the prediction of the usual BCS model, $8.5 e^{-1.44\left(T_{c} / T\right)}$, for $2.5<T_{c} / T<6$. Theoretical plots for $q=1$ and 0.99 agree well to the experimental fitting above. However, one can see in Fig. 1, specially in the insert, that the agreement for $\mathrm{V}$ with $q=0.99$ is better not only at $T \sim T_{c}$, but specially for lower temperatures. At such low temperatures there is an increasing departure from the standard statistics. 


\section{Table 1}

\begin{tabular}{|c||c|c|}
\hline & $\left.\frac{C_{e}^{(s)}-C_{e}^{(n)}}{C_{e}^{(n)}}\right|_{T_{c}}$ & $q$ \\
\hline \hline $\mathrm{Al}$ & $1.29-1.59$ & $1.03-0.996$ \\
$\mathrm{Zn}$ & 1.3 & 1.03 \\
$\mathrm{In}$ & 1.73 & 0.993 \\
$\mathrm{Mo}$ & 1.28 & 1.03 \\
$\mathrm{Nb}$ & 1.87 & 0.975 \\
$\mathrm{Sn}$ & 1.6 & 0.995 \\
$\mathrm{Ta}$ & 1.59 & 0.995 \\
$\mathrm{Tl}$ & 1.5 & 0.997 \\
& & \\
\hline
\end{tabular}

Display of the values $q$ found when numerical plot is compared to the specific heat jump measured experimentally $[22] . C_{e}^{(s)}$ was calculated numerically in the weakcoupling limit. Notice that these usual superconductors are well described by $q \approx 1$.

By the same token, on Fig. 2 we show several data for the specific heat for $\mathrm{Al}$ and Zn. They both deviate from the usual BCS calculations in the low temperature regime. The data of Phillips[25] for $\mathrm{Zn}$ is better fitted by a $T^{3}$ law in the low temperature regime. According to our estimations, shown in Table 4.1, the $\mathrm{Al}$ and $\mathrm{Zn}$ data should be fitted by $q=1.03$. Indeed, one can see in Table 4.1 that the calculations with $q=1.03$ agree much better with the experimental results of several groups than the usual statistics. We can also see in Fig. 2 the tendency of the specific heat to deviate from a exponent to a power law behavior even with such small value of $q=1.03$. This behavior is even stronger with a much larger value of $q$, like $q=1.2$ for instance, although such calculations will be presented elsewhere.

\subsection{Ultrasonic Attenuation Rate}

In this section we treat the problem of acoustic waves perturbing the electronic system described by the diagonalized BCS reduced Hamiltonian. We will consider only longitudinal waves and the temperature interval, the frequency and wave vector are related by the inequality $w<v_{F} p<\Delta / \hbar$. As usual, we assume that the matrix element of the perturbation depends on $\mathbf{k}^{\prime}-\mathbf{k}$. In a typical ultrasound experience the frequency is less than $10^{9} \mathrm{~Hz}$, hence $\hbar w \ll k_{B} T$, 
and in the limit of very low frequencies one get that the normalized ultrasonic attenuation rate within Tsallis' statistics is

$$
\frac{\alpha_{s}}{\alpha_{n}}=-\int_{-\infty}^{\infty} \frac{\partial f_{q}}{\partial E}=2 f_{q}(\Delta)
$$

Notice that $\alpha_{s} / \alpha_{n}$ also has exponential decrease when $q \rightarrow 1$ in the low temperature regime. Such behavior is experimentally verified in several usual superconductors, although plots of experimental data tend to fall off somewhat more rapidly just below $T_{c}$. Data of Morse and Bohm[29]for Sn are a example, Fig.3, and are compared with theoretical plots for $q=0.99$ and 1. Again, $q=0.99$ is in better agreement with experimental results. An interpretation for the discrepancy between the usual theory and the data was given in terms of the gap function, which, compared with it value at $T=0$, rises slightly too rapidly as the temperature decreases just below $T_{c}$. Tunneling measurements for Sn by Morse and Bohm[30] are consistent with such feature. On Fig. 4 we show their results for Sn and In with the calculation with $q=0.99$ and 1 . We can easily see that $q=0.99$ is in much better agreement than the usual $q=1$ results. Thus in the present interpretation this result is attributed to the nonextensivity of the system.

\section{Conclusions}

We conclude this paper pointing out our new findings:

- We have show that the BCS potential widely used in the description of superconductors is nonextensive. Following this result we have developed a BCS theory with nonextensive entropy which depends on the index $q$. We have, therefore, derived some thermodynamic quantities that can be compared with the experiments.

- Using the experimental values of the specific heat, we estimated the nonextensive index $q$ for several superconductors. Explicit calculations with such values of $q$ for several materials are indeed in much better agreement with the present experimental results than the usual values. In particular for Sn we have estimated $q \approx 0.99$ and have shown that with such value we reproduced with a better agreement the experimental results of the specific heat, ultrasonic attenuation and tunneling experiments. Similar calculations were performed for $\mathrm{Al}$ and $\mathrm{Zn}$. The fact that three well-known properties are fitted by the same $q \neq 1$ rules out a possible deviation due to the approximations on the calculations. 
Furthermore, we have seen that as $q$ increases, the exponential behavior of the specific heat changes in a power law behavior. This is a novel result and it means that even if there is a constant gap in the neighborhood of a Fermi surface, we can get a power law behavior once we use Tsallis statistics with $q \neq 1$. Several new superconductors, like high temperature superconductors for instance, exhibit such power law behavior which is attributed to a non-constant d-wave order parameter. At the moment we are developing a non weak-coupled theory of superconductivity with Tsallis statistics to deal with these and others exotic superconductors with thermodynamical quantities which exhibit a power law behavior.

\section{ACKNOWLEDGMents}

We thank Conselho Nacional de Desenvolvimento Científico e Tecnológico-CNPq-Brasil e FAPERJ for partial financial support.

\section{References}

[1] Constantino Tsallis, J. Stat. Phys. 52, 479 (1988).

[2] Brazilian Journal of Physics 29 , N.1, March 1999.

[3] A. R. Plastino, A. Plastino, Phys. Lett. A A4, (1990), 384.

[4] G. Kaniadakis, A. Lavagno, P. Quarati Phys. Lett. B319, (1996), 308.

[5] A. Lavagno, G. Kaniadakis, M. R. Monteiro, P. Quarati, C. Tsallis, Astrophys. Lett. and Comm. 35, (1998), 449.

[6] A. Plastino and C. Tsallis, J. Phys. A: Math. Gen. 26, L893-L896 (1993).

[7] A. Plastino and A. R. Plastino, Physics Letters A 226, 257-263 (1997).

[8] D. Prato, Phys. Lett. A 203, 165-168 (1995).

[9] A. K. Rajagopal, R. S. Mendes and E. K. Lenzi, Phys. Rev. Lett. 80, 3907 (1998).

[10] E. K. Lenzi, R. S. Mendes and A K. Rajagopal, Quantum Statistical Mechanics for Nonextensive Systems II, preprint.

[11] E. M. F. Curado and C. Tsallis, J. Phys. A: Math. Gen. 24, L69-L72 (1991).

[12] Fevzi Büyükkılıç, Dogan Demirhan and Atilla Güleç, Phys. Lett. A 197, 209220 (1995).

[13] Exact and approximate results of nonextensive quantum statistics, preprint.

[14] J. Bardeen, L. N. Cooper and J. R. Schrieffer, Phys. Rev. 108, 1175 (1957). 
[15] P. W. Anderson, Phys Rev. 112, 1900 (1958).

[16] N. Bogoljubov, J. Phys USSR 11, 22 (1947).

[17] J. R. Schrieffer, Theory of Superconductivity , Addison-Wesley, New York, 1964.

[18] M. Tinkham, Introduction to Superconductivity, McGraw-Hill, NY, 1975.

[19] C. Anteneodo and C. Tsallis, Phys. Rev. Lett. 80, 5313 (1998).

[20] N. N. Bogolubov and N. N. Bogolubov, Jr., Introduction to Quantum Statistical Mechanics, World Scientific, 1982.

[21] R. D. Mattuck, A Guide to Feynman diagrams in the many-body problem, p. 386, McGraw-Hill, NY, 1976.

[22] R. Meservey and B. B. Schwartz, Equilibrium Properties: Comparison of Experimental Results with Predictions of the BCS Theory, in Superconductivity, edited by R. D. Parks, Marcel Dekker, Inc. (1969).

[23] H. A. Boorse, Phys. Rev. Lett. 9, 391 (1959).

[24] B. B. Goodman, Comp. rend. 244, 2899 (1957),

[25] N. E. Phillips, Phys. Rev. Lett. 1, 363 (1958).

[26] M. A. Biondi, A. T. Forrester, M. P. Garfunkel, and C. B. Satterthwaite, Rev. Mod. Phys. 30, 1109 (1958).

[27] N. V. Zavaritskii, JETP 7, 773 (1958).

[28] W. S. Corak, B. B. Goodman, C. B. Satterwhaite, and A. Wexler, Phys. Rev. 96, 1442 (1954).

[29] R. W. Morse and H. V. Bohm, Phys. Rev. 108, 1094 (1957).

[30] I. Giaever and K. Megerle, Phys. Rev. 122, 1101 (1961). 
Fig. 1. Comparison between the fitting of the low temperature electronic specific heat of Tin, $C_{e}^{(s)}=9.17 \exp \left[-1.5\left(T / T_{c}\right)\right]\left[\gamma T_{c}\right]$, and the theoretical plots for $q=1$ and 0.99. Both values of $q$ describe very well the experimental results, but for $\mathrm{V}$ in particular, regarding the interval of temperature $0.25<T / T_{c}<0.7$, a better agreement was found with $q=0.99$.

Fig. 2. Experimental data for the specific heat of $\mathrm{Al}$ and $\mathrm{Zn}$ [23]. Notice that the measurements by Phillips[25], Goodman[24] e Zavaritskii[27] for the Aluminum are well described by the generalized BCS model with $q=1.03$, the same value furnished in Table 4.1. Also notice that data obtained by Phillips[25] for Zinc are very well described by $3\left(T_{c} / T\right)^{3}$ in the low temperature regime.

Fig. 3. Ultrasonic attenuation rate calculated for different values of $q$ and compared to experimental data for $\operatorname{Sn}[29]$. A better agreement was found with $q=0.99$, as proposed by measurements of the specific heat jump.

Fig. 4. Comparison between the gap energy from tunneling measurements for Tin and Indium[30] and theoretical plots using $q=1$ and 0.99 . Notice that the gap function related to $q=0.99$ have better qualitative agreement with experimental results. 
This figure "Fig1.jpg" is available in "jpg" format from: http://arxiv.org/ps/cond-mat/0108251v1 
This figure "Fig2.jpg" is available in "jpg" format from: http://arxiv.org/ps/cond-mat/0108251v1 
This figure "Fig3.jpg" is available in "jpg" format from: http://arxiv.org/ps/cond-mat/0108251v1 
This figure "Fig4.jpg" is available in "jpg" format from: http://arxiv.org/ps/cond-mat/0108251v1 